\title{
Le ditax (Detarium senegalense J. F. Gmel.) : principales caractéristiques et utilisations au Sénégal
}

\author{
Nafissatou DIOP ${ }^{1}$, Augustin NDIAYE ${ }^{1}$, Mady CISSE $^{2}$, Oumar DIEME $^{1}$, Manuel DORNIER ${ }^{3 *}$, Oumar SOCK ${ }^{2}$
}

${ }^{1}$ Inst. Technol. Aliment., Route des Pères Maristes, BP 2765, Dakar - Hann, Sénégal

2 ESP, Univ. Cheikh Anta Diop, BP 5085, Dakar - Fann, Sénégal

${ }^{3}$ Cirad, Persyst, Montpellier SupAgro, UMR 95 Qualisud, TA B-95/16, 34398 Montpellier cedex 5, France, dornier@cirad.fr
* Correspondance et tirés à part

Reçu le 1 décembre 2009 Accepté le 4 mars 2010

Fruits, 2010, vol. 65 , p. 293-306 (C) 2010 Cirad/EDP Sciences All rights reserved DOI: $10.1051 /$ fruits/2010025 www.fruits-journal.org

RESUMEN ESPAÑOL, p. 306
The ditax (Detarium senegalense J.F. Gmel.): main characteristics and uses in Senegal.

Abstract - Botany. Originating from tropical Africa, Detarium senegalense J.F. Gmel. is a multicaulous big tree. The height varies between $(15$ and 40$) \mathrm{m}$. It belongs to the Caesalpiniaceae family and the Detarium Juss. genus. Its leaves are paripinnate, with five to six pairs of opposite leaflets laid out in an alternate manner. Distribution. D. senegalense is found on the wet dense edges of forests, in the coastal and northern regions, and the Sudan-Guinean zone. Also called "ditax" in Wolof, D. senegalense belongs to the most important forest fruit-bearing species in the economy of exploitation in Senegal. It grows in the wild in the Sine-Saloum islands and in Casamance. Composition and processing. The fruit is characterized by its green pulp which is very rich in vitamin C (more than $1 \mathrm{~g} \cdot 100 \mathrm{~g}^{-1}$ ). It is a very popular fruit and its pulp is widely consumed in Senegal as nectar, marmalade and sherbets, or directly eaten. Moreover, the fruit also has therapeutic virtues, such as cough treatment, kidney pain relief and leprosy treatment. Some varieties of $D$. senegalense produce toxic fruits but the compounds responsible for the toxicity have not been completely identified yet. Up to now, there is no objective method to differentiate edible fruits from toxic fruits. Assessment. In spite of its nutritional and organoleptic potential, ditax remains under-exploited. Consequently, a complete biochemical characterization of fruits, evaluation of technological potential, development of methods for a rapid detection of toxic fruits, and stability improvement of ditax processed products would be interesting research prospects for a better valorization of this fruit, which is rich in vitamin C. The domestication of the plant also deserves consideration for development of the production.

Senegal / Detarium senegalense / fruits / proximate composition / traditional uses / ingestion toxicity / ascorbic acid

Le ditax (Detarium senegalense J. F. Gmel.) : principales caractéristiques et utilisations au Sénégal.

Résumé - Botanique. Originaire d'Afrique tropicale, Detarium senegalense J.F. Gmel. appartient à la famille des Caesalpiniaceae et au genre Detarium Juss. C'est un grand arbre multicaule de (15 à 40) m de haut. Ses feuilles sont composées, paripennées, avec cinq à six paires de folioles opposées, disposées de manière alterne. Répartition. D. senegalense est rencontré sur les lisières de la forêt dense humide, dans les régions côtières, septentrionales et dans la zone soudano-guinéenne. Désigné par le terme " ditax " en Wolof, D. senegalense fait partie des espèces fruitières forestières les plus importantes dans l'économie d'exploitation au Sénégal. Il pousse de façon sauvage dans les îles du Sine-Saloum et en Casamance. Composition et transformation. Le fruit est caractérisé par sa pulpe verte très riche en vitamine $C$ (plus de $1 \mathrm{~g} \cdot 100 \mathrm{~g}^{-1}$ ). C'est un fruit très populaire et largement consommé au Sénégal principalement sous forme de boisson, de marmelade, de sorbet ou à l'état frais. Le fruit possède également des vertus thérapeutiques contre la toux, les maux de reins et la lèpre. Certaines variétés de $D$. senegalense produisent des fruits toxiques mais les composés responsables de la toxicité n'ont pas encore été totalement identifiés. Aucune méthode objective permettant de différencier les fruits comestibles des fruits toxiques n'est disponible à l'heure actuelle. Bilan. Malgré ses potentialités nutritionnelles et organoleptiques, le ditax reste sous-exploité. Dès lors, la caractérisation biochimique complète des fruits, l'évaluation du potentiel technologique, le développement de méthodes de détection rapide des fruits toxiques et l'amélioration de la stabilité des produits transformés à base de ditax seraient des perspectives de recherches intéressantes pour mieux valoriser ce fruit riche en vitamine C. La culture contrôlée de la plante mériterait également d'être envisagée pour le développement de la production.

Sénégal / Detarium senegalense / fruits / composition globale / utilisation traditionnelle / toxicité par ingestion / acide ascorbique 


\section{Introduction}

Originaire d'Afrique tropicale, Detarium senegalense J.F. Gmel. appartient au genre Detarium qui a été décrit pour la première fois en 1789, sous le nom de "Detar " du Sénégal par De Jussieu dans le Genera Plantarum [1]. Cependant, c'est à Guillemin auteur de l'édition XIII du Systema Naturea (1788-1793) qu'on doit la nomenclature binaire du "detah": Detarium senegalense J. F. Gmel., ce nom provenant de "Ditah " dont Adanson a fait emprunt aux Sénégalais dans son manuscrit [2-4]. En effet, le premier échantillon de Detarium fut récolté au Sénégal par Adanson entre 1749 et 1753 [3]. Plusieurs auteurs se sont par la suite intéressés au genre Detarium à cause de la mise en évidence de deux variétés présentant des morphotypes difficiles à différencier, dont l'une produisait des fruits toxiques. Ce fait rare également observé chez l'espèce $A m y g$ dalus communis L., dont deux variétés contiennent à des doses différentes des hétérosides cyanogènes, est à l'origine de la confusion dans l'identification des espèces de Detarium [4].

Au Sénégal, D. senegalense J.F. Gmel. fait partie des espèces fruitières forestières les plus importantes dans l'économie d'exploitation et l'économie nationale. L'arbre pousse de façon sauvage en Casamance et dans les Îles du Sine-Saloum. Le fruit du D. senegalense, appelé " ditax " ou " ditakh " en Wolof, est très populaire et largement consommé au Sénégal principalement sous forme de boissons, de marmelades, de sorbets et à l'état frais. Cependant, le fruit reste

Figure 1.

Detarium senegalense J. F. Gmel dans son habitat naturel (île de Falia, Sénégal).

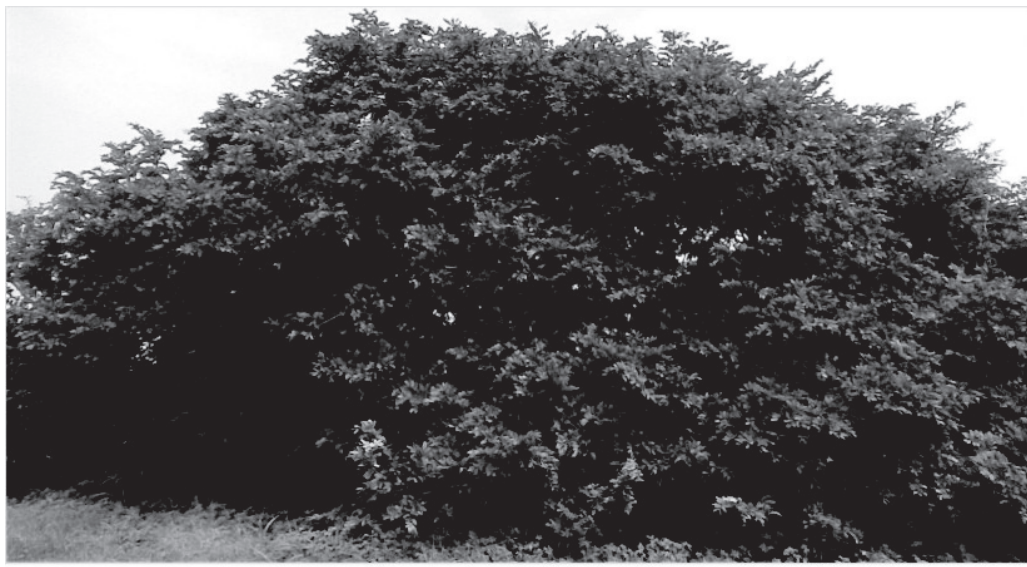

sous-valorisé dans son ensemble avec d'importantes pertes post-récolte. La caractérisation biométrique, physico-chimique, biochimique et aromatique du fruit pourrait contribuer significativement à enrichir les connaissances et à comprendre les problèmes liés à la stabilité des produits issus de la transformation du fruit.

Notre travail bibliographique s'intègre dans le cadre d'un projet intitulé "Amélioration de la compétitivité des boissons et nectars à base de fruits locaux au Sénégal ", financé par le Fonds National de Recherche Agricole et Agroalimentaire du Sénégal (FNRAA). Il se propose de faire la compilation des informations disponibles dans la littérature et des résultats de recherches effectuées à l'Institut de Technologie Alimentaire (ITA) de Dakar sur les principales caractéristiques et utilisations du ditax comestible au Sénégal afin de contribuer à l'évaluation pertinente de l'intérêt de ce fruit très riche en vitamine $\mathrm{C}$.

\section{Botanique}

Detarium senegalense J.F. Gmel. appartient à l'embranchement des spermaphytes, au sous-embranchement des angiospermes, à la classe des dicotylédones, à la sous-classe des dialypétales, à la série des caliciflores, à l'ordre des rosales, au sous-ordre des légumineuses, à la famille des Caesalpiniaceae et au genre Detarium Juss. [4].

Detarium senegalense est un grand arbre multicaule de (15 à 30) $\mathrm{m}$ de haut pouvant atteindre parfois $40 \mathrm{~m}$ dans les galeries forestières. Sa cime est dense et hémisphérique, ses branches sont trapues et étalées (figure 1). Le fût est droit. L'écorce (figure 2) est épaisse, grisâtre parfois bleutée, légèrement fissurée et écailleuse chez les gros arbres. Les feuilles (figure 2) sont composées paripennées avec 5 à 6 paires de folioles opposées disposées de manière alterne. Les folioles sont longues de (4 à 6) cm et larges de (3 à 4) cm, ovales à elliptiques, arrondies aux extrémités et émarginées au sommet. Le limbe est mince, souple, finement coriace, à nervation pennée et vert sur le dessous. L'inflorescence est en panicule axillaire plus ou moins ramifiée et 
longue de (5 à 8) cm (figure 2). Les fleurs blanc crème, en racème, ont un calice glabre ou glabrescent. Elles présentent quatre sépales elliptiques avec un ovaire ovoïde et pubescent, et dix étamines blanches biloculaires [4, 5-12].

\section{A propos de la forme toxique du ditax}

En décrivant l'espèce Detarium senegalense, Guillemin et al. (1830-1833) ont observé que cet arbre pouvait produire des fruits comestibles ou toxiques [13]. Les cas d'intoxication, avec parfois des décès enregistrés à la suite de l'ingestion des fruits de certains arbres de cette espèce, ont conduit plusieurs auteurs à reconnaître l'existence de deux variétés : la variété à fruits comestibles et la variété à fruits toxiques [4, 14]. Ainsi Baillon décrivit entre 1865-1866 l'arbre produisant des fruits toxiques en tant qu'espèce distincte qu'il nomma Detarium beudelotianum Baill. [15]. Pour désigner le fruit toxique, certains auteurs parlent de "variété toxique de 'ditakh' " [16, 17], de " faux detah " [18], de " fruit toxique du Detarium " [19] ou alors de " variété à fruits amers de Detarium senegalense " [20, 21]. D'autres auteurs parlent de "variété à fruits toxiques de Detarium senegalense " [22, 23]. Cependant, la question du rang taxonomique de la forme toxique est encore d'actualité avec beaucoup de controverses dans l'identification des formes toxiques et comestibles. En effet, certains auteurs considèrent qu'il s'agit de deux variétés différentes, tandis que d'autres estiment qu'il s'agit simplement de formes différentes.

Sambuc (1887) de même qu'Heckel et Schlagdenhauffen (1889) étudièrent les fruits réputés toxiques sans mettre en évidence de principe vénéneux [19, 20]. Par la suite, Aubreville et Trochain (1937) considérèrent quant à eux que $D$. heudelotianum était le synonyme de Detarium senegalense, ce dernier comportant deux variétés : l'une à fruits comestibles, l'autre à fruits amers [24]. Pour Paris et Moyse-Mignon (1947), les fruits toxiques appartiendraient à l'espèce $D$. heudelotianum alors que les fruits

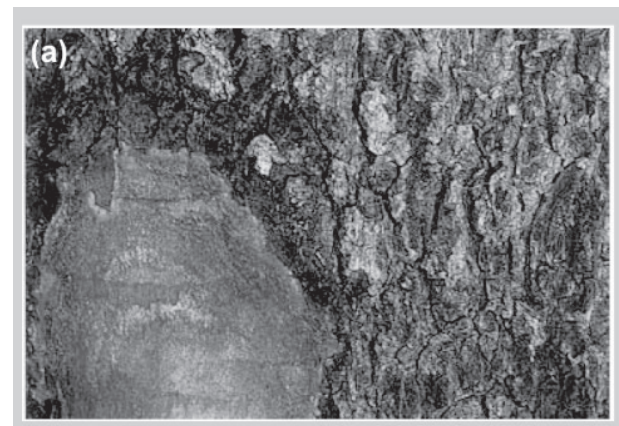

Figure 2.

Ecorce (a), folioles (b) et fleurs (c) de Detarium senegalense J. F. Gmel. [5].
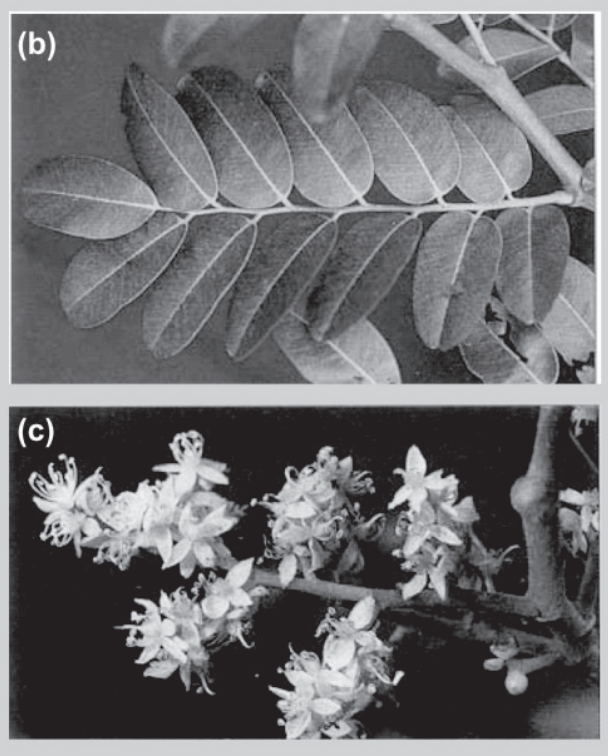

comestibles correspondraient à l'espèce D. senegalense [18]. Cependant, des études récentes menées par Cavin (2007) apportent des clarifications intéressantes sur la question du rang taxonomique [6]. En effet, suite à ses travaux, l'auteur considèrerait l'existence d'une espèce de Detarium senegalense avec deux variétés, l'une produisant des fruits toxiques et l'autre des fruits comestibles. Dans ces mêmes travaux, l'auteur a mis en évidence un dérivé glycosidique cyanogène, la 6'-O-galloyl- $(R)$-épihétérodendrine (figure 3), qui ne serait présent que dans la variété à fruits toxiques ; cela le conduit à conclure qu'il s'agirait du, ou d'un des, composé(s) responsable(s) de la toxicité. La forme toxique se différencie par des folioles souples, vert vif dessus et vertes dessous, et par une pulpe légèrement plus fibreuse [6]. 
Figure 3.

Structure du composé G : 6'O-galloyl-(R)-

épihétérodendrine, dérivé glycosidique cyanogène qui ne serait présent que dans la variété à fruits toxiques de Detarium senegalense J. F. Gmel. [6].

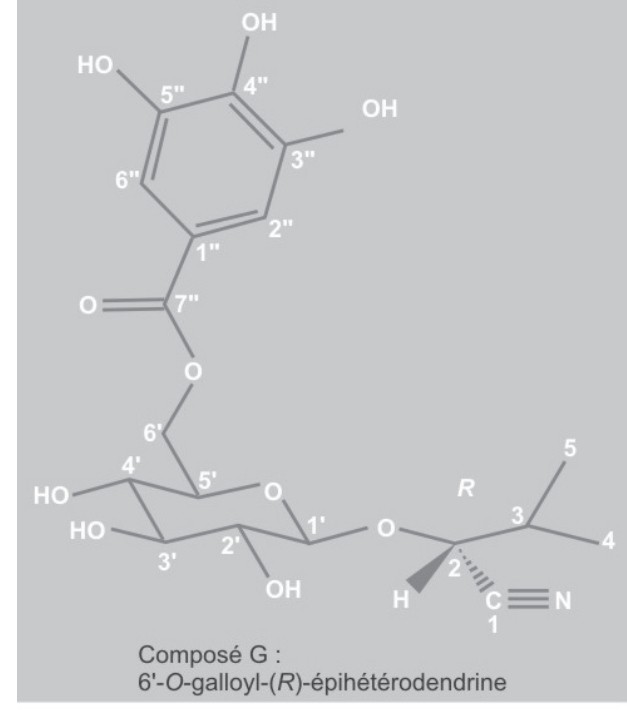

Actuellement, au Sénégal, l'innocuité des fruits commercialisés repose uniquement sur la connaissance des populations locales qui distinguent les deux formes sur la base de l'amertume et de l'odeur caractéristique des fruits toxiques, ainsi que par la présence de nombreux fruits intacts tombés au pied des arbres et non consommés par les animaux, notamment par les singes et les oiseaux [14, 21]. Par cette technique, les fruits toxiques ne sont pas récoltés; de ce fait, les produits dérivés et en particulier le nectar de ditax ne sont pas concernés. Les cas d'intoxication sont dus à la consommation, au niveau de sites de récolte, de fruits toxiques seuls. Ce serait l'explication de huit cas d'intoxication qui ont été enregistrés au service de pédiatrie de l'hôpital principal de Dakar (Sénégal) [14]. Les symptômes observés au cours de ces intoxications sont variables (tableau I). Ils apparaissent brutalement (1 à 6) h après l'ingestion. Ils ne sont pas spécifiques et varient en fonction des patients.

Par ailleurs, des enquêtes effectuées dans les deux principales zones de production du ditax au Sénégal, les régions de Fatick et de Ziguinchor, ont montré que la variété toxique ne se trouvait que dans la région de Ziguinchor, et cela dans des sites bien précis [25]. Il semblerait aussi que les arbres à fruits toxiques soient de moins en moins présents car éliminés par les populations locales.

\section{Aires de répartition et noms vernaculaires}

En Afrique, D. senegalense se répand sur les lisières de la forêt dense humide, dans les régions côtières et dans les régions septentrionales de Côte-d'Ivoire et pénètre ainsi dans la zone soudano-guinéenne, depuis le Soudan, la Guinée-Conakry, jusqu'en Afrique centrale [26]. L'arbre pousse sur les basfonds humides et les sols frais ; elle est présente en Gambie, Guinée, Sierra Leone, Liberia, Côte-d'Ivoire, Ghana, Nigeria, Cameroun, République centrafricaine et Mozambique $[5,27]$. L'espèce $D$. senegelasense est de plus en plus retrouvée dans la forêt tropicale humide guinéo-congolaise où la pluviométrie est plus élevée que dans la zone soudano-guinéenne [6].

Au Sénégal, ce sont particulièrement les régions de Ziguinchor et de Fatick (îles du Sine et du Saloum) qui constituent actuellement les principales zones de production (figure 4) du D. senegalense [7]. Dans la région de Fatick, les zones de production sont : Fatick, Tataguine, Niodior, Dionewar, Falia, Foundiougne, Betenti, Sokone et

Tableau I.

Symptômes observés suite à l'ingestion du fruit toxique de Detarium senegalense J. F. Gmel. [6, 14, 16, 23].

\begin{tabular}{|c|c|c|c|c|c|}
\hline Personnes cibles & $\begin{array}{c}\text { Système } \\
\text { cardiovasculaire }\end{array}$ & $\begin{array}{l}\text { Système } \\
\text { digestif }\end{array}$ & $\begin{array}{l}\text { Système } \\
\text { respiratoire }\end{array}$ & $\begin{array}{c}\text { Système } \\
\text { nerveux central }\end{array}$ & $\begin{array}{c}\text { Examen } \\
\text { de laboratoire }\end{array}$ \\
\hline $\begin{array}{l}\text { Tout âge et des deux } \\
\text { sexes, avec une } \\
\text { fréquence plus élevée } \\
\text { chez les enfants }\end{array}$ & $\begin{array}{c}\text { Accélération du pouls } \\
\text { Hypothermie } \\
\text { Hypotension }\end{array}$ & $\begin{array}{c}\text { Dhiarrhées } \\
\text { Vomissement } \\
\text { Douleur abdominale }\end{array}$ & $\begin{array}{l}\text { CEdème } \\
\text { pulmonaire } \\
\text { aigu }\end{array}$ & $\begin{array}{c}\text { Somnolence } \\
\text { Convulsion associée à } \\
\text { une mydriase } \\
\text { bilatérale }\end{array}$ & $\begin{array}{l}\text { Hyperglycémie } \\
\text { Hypoglycémie } \\
\text { Vitesse de } \\
\text { sédimentation } \\
\text { accélérée }\end{array}$ \\
\hline
\end{tabular}




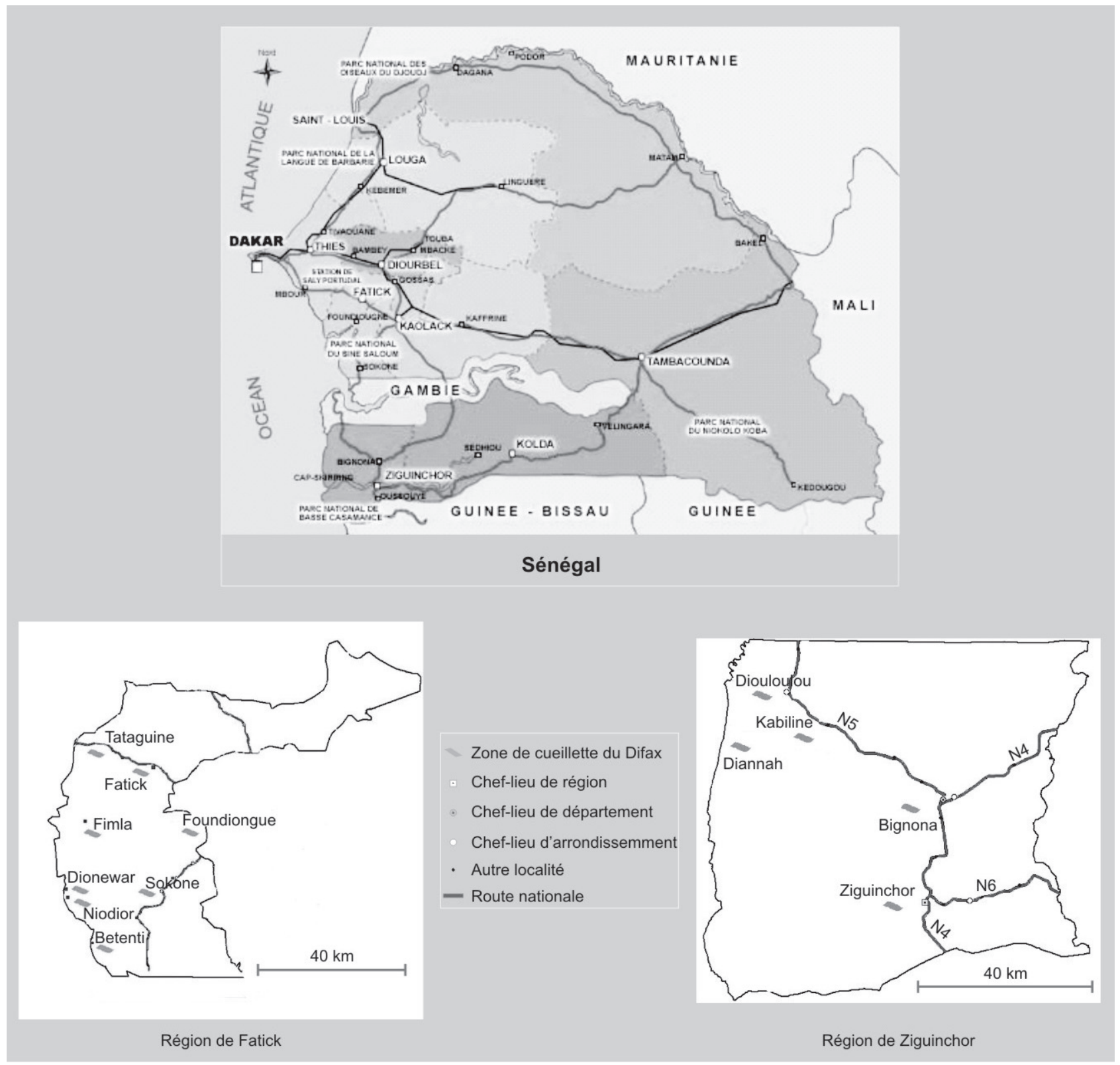

Fimla [25]. La floraison y commence entre mars et avril et les fruits mûrissent d'août à novembre. Le ditax est retrouvé dans la zone en groupe ou éparpillé dans les champs. Dans la région de Ziguinchor, D. senegalense est observé à Diouloulou, kabiline, Bignona et Diannah. L'espèce est également présente en individus très dispersés dans les environs de Dakar, à proximité des Niayes et remonte le littoral vers le nord dans le sahel côtier.
Detarium senegalense préfère les lieux Figure 4.

frais des savanes humides et les galeries Principales zones de cueillette forestières. Il est présent dans la partie ouest du ditax (Detarium senegalense du climat sahélo-soudanien caractérisé par J. F. Gmel.) dans les régions de un climat d'alizés maritimes avec une tem- Fatick et de Ziguinchor au pérature moyenne annuelle de $26-31^{\circ} \mathrm{C}$. Sénégal (d'après le site http:// C'est un climat sec avec des variations consi- www.gouv.sn/IMG/jpg/cartedérables d'humidité. La saison des pluies sen.jpg, consulté le 2 avril dure de 2 à 4 mois avec un maximum de 2010). une moyenne annuelle de (400 à 1200) mm. 
Tableau II.

Appellations vernaculaires de Detarium senegalense J.F. Gmel au Sénégal [4, 9-11, 18, 22, 24, 27, 28].

\begin{tabular}{lcc}
\hline Dialecte & Variété à fruits comestibles & Variété à fruits toxiques \\
\hline $\begin{array}{l}\text { Baïnouk } \\
\text { Balante }\end{array}$ & Si moukat, sou moukat, si onni, ki bodé, boupokoten & - \\
Bambara & Blanti, blundi, blundi pok & - \\
Bassari & Tamba, tambacounda & - \\
Diola & Atiackechs & Bou foulounkat \\
Diola fogny & Bou gnagnoud, bou bounkout & - \\
Foula & Boubounkoutabou, bougoungout, bounoun & - \\
Français & Kérèndouta & Faux detar \\
Malinké & Grand detar & Bodo \\
Mandingue & Tamba, talo, sankosémou & - \\
Peul & Mamboda, mambodo, saroko, tali dima & - \\
Sérère & Mbodéy, mobodéy, bodo, datékéi, dolé & Ndali \\
Sérère none & Ndoy, ndogoy, ndoroy & - \\
Sérère safène & Tangalang & - \\
Socé & Xoum & Tali, talo \\
Wolof & Ditax & Talèm baro, tali kougna
\end{tabular}

Ce climat est caractéristique du littoral du Sénégal, de la Gambie, de la Basse-Casamance et de la Guinée-Bissau. On retrouve l'espèce dans la partie ouest du climat soudano-guinéen avec une température moyenne annuelle de $24-28^{\circ} \mathrm{C}$ et une pluviométrie moyenne annuelle de (950 à 1750) $\mathrm{mm} \mathrm{[7].}$

Au Sénégal, les fruits comestibles et toxiques de $D$. senegalense portent des noms vernaculaires qui varient selon le dialecte parlé par les populations locales (tableau II)

\section{Le fruit}

\subsection{Généralités}

Sur le plan botanique [5, 7, 10, 11], le Ditax est une drupe globuleuse ou subglobuleuse, aplatie, de (3 à 8) $\mathrm{cm}$ de diamètre (figure 5a). Le fruit est constitué de trois parties principales : 1/ l'épicarpe vert foncé, dur pour les fruits immatures, vert clair tirant sur le marron et cassant pour les fruits mûrs (figure 5b), 2/ le mésocarpe verdâtre (pulpe) entremêlé de fibres insérées sur le noyau correspondant à la portion comestible du fruit qui représente environ $45 \%$ de sa masse et 3/ le noyau (figure 5c) volumineux, ligneux recouvert de mailles fibreuses et renfermant une seule graine ovoïde et aplatie de couleur marron foncé, qui représente également $45 \%$ de la masse du fruit [5, 7, 11, 12, 29-31].

Entre deux phases de fructification, l'arbre subit généralement une défeuillaison totale qui intervient fin février-début mars. La nouvelle feuillaison suivie de la floraison s'étale de mars à juin selon que la variété est précoce ou tardive et en fonction des zones. Les fruits sont à maturité entre août et septembre pour les régions de Fatick et Kaolack ; entre novembre et décembre pour les régions de Ziguinchor et Kolda [7, 11, $12,25]$.

\subsection{Stade de récolte optimal et durée de conservation}

Des travaux préliminaires menés sur deux sites de production au Sénégal (îles de Djilor et de Mar Fafaco) ont montré que le ditax pouvait mûrir sur l'arbre entre (170 et 200) j après la nouaison, soit entre (6 et 7) mois [29, 31]. Les fruits récoltés au bon stade de développement peuvent subir aussi une maturation complémentaire en utilisant du carbure de calcium ou de l'azéthyl. A ce stade, le calibre des fruits peut varier entre 
( 4 et 4,5$) \mathrm{cm}$ de longueur et (3 à 5) $\mathrm{cm}$ de diamètre $[10,11,29,31]$. Au cours du développement, l'acidité diminuerait alors que les sucres et la vitamine $\mathrm{C}$ augmenteraient. Les fruits qui n'ont pas un développement suffisant ne mûrissent pas quelle que soit la méthode de récolte utilisée. La récolte précoce des fruits immatures entraîne des pertes post-récolte importantes car les fruits sont totalement déformés et doivent être jetés. Les fruits récoltés au bon stade de maturité se détériorent si certaines précautions ne sont pas prises [29, 31]. Pour la conservation, la méthode traditionnelle consiste à sécher les fruits mûrs, puis à les décortiquer. Ils sont ensuite conditionnés dans des paniers en feuilles de rônier qui permettent une aération partielle [31]. L'entreposage des fruits frais à $4{ }^{\circ} \mathrm{C}$ permet de conserver le ditax entre ( 45 et 60 ) j avec des pertes en vitamine $\mathrm{C}$ de l'ordre de $6 \%$, une perte de masse de $5 \%$ et un taux d'altérations fongiques de $7 \%$. Lorsque l'intégrité de la coque est altérée, des phénomènes d'oxydations apparaissent très rapidement et la couleur verte caractéristique de la pulpe change vers le brun jaunâtre [29, 31].

\subsection{Composition chimique et utilisations}

La première étude biochimique des fruits du ditax a été effectuée en 1887 par Sambuc [19]. Déjà en 1948, Auffret notait une teneur en vitamine $\mathrm{C}$ de $2 \mathrm{~g} \cdot 100 \mathrm{~g}^{-1}$ de pulpe, ce qui classerait le ditax parmi les fruits de cueillette le plus riche en vitamine C [32]. En 1967, Toury et al. avaient également trouvé $1290 \mathrm{mg}$ de vitamine $\mathrm{C} \cdot 100 \mathrm{~g}^{-1}$ de pulpe [33]. Globalement, la pulpe de ditax se caractériserait par une teneur en vitamine C supérieure à $1000 \mathrm{mg} \cdot 100 \mathrm{~g}^{-1}$ de pulpe fraîche $[4,10,30,34,35]$ (tableau III).

L'acidité du fruit $\left(0,16 \mathrm{mEq} \cdot 100 \mathrm{~g}^{-1}\right)$ est relativement faible [36]. La pulpe renfermerait également des polyphénols, notamment de l'acide gallique, [11] et présenterait des trace d'alcaloïdes, de saponines et de tanins dont la structure n'a cependant pas été déterminée [23].

L'identification des composés d'arômes de la pulpe de ditax a révélé la présence

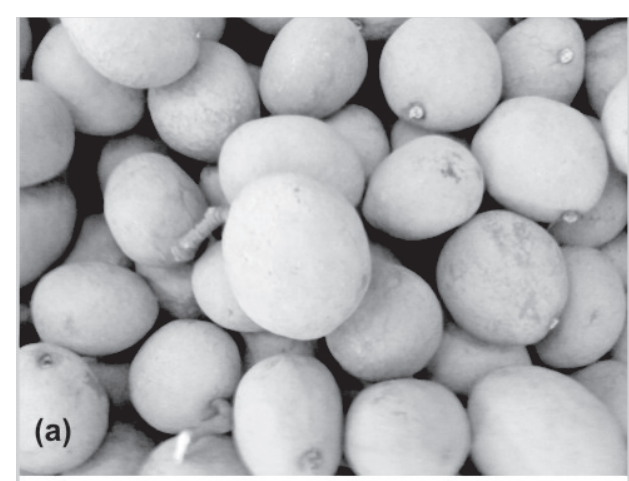

Figure 5.

Fruits (a), épicarpe (coques) (b) et noyau dépulpé (c) [11] de Detarium senegalense J.F. Gmel.
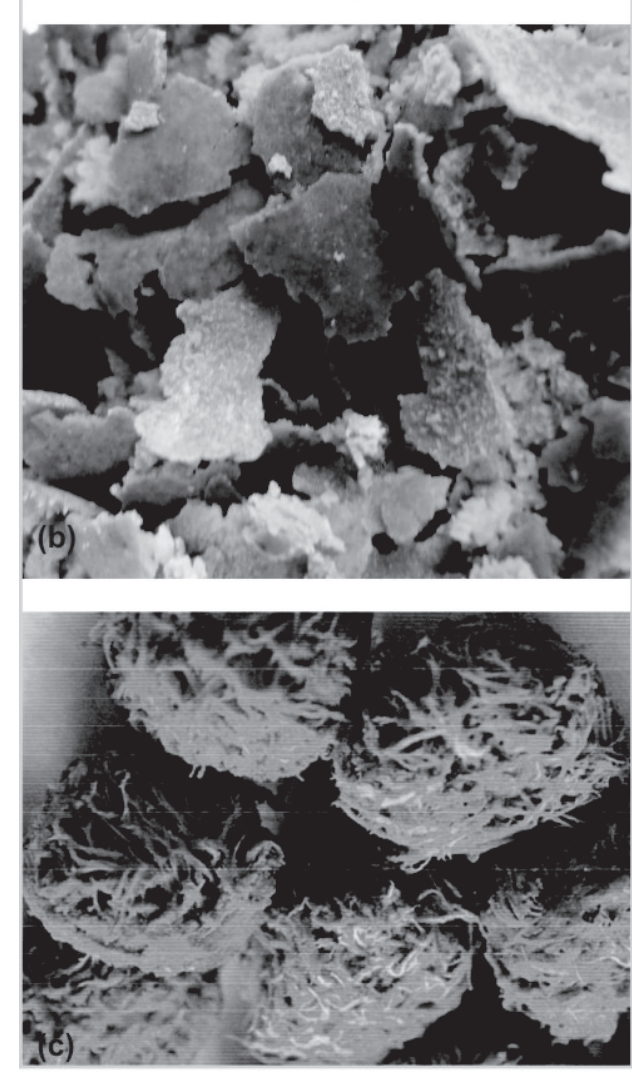

d'alcools supérieurs (méthyle-buténol, penténol, hexanol) et de dérivés en $\mathrm{C}_{6}$ (aldéhydes et alcools issus du catabolisme des lipides via une lipoxygénase) dont les caractéristiques olfactives présentent des notes herbacées prononcées. Une forte présence de notes de melon/concombre a été notée de même que la présence d'alcools et d'aldéhydes en $\mathrm{C}_{7}$ responsables de l'arôme du ditax [31].

Le fruit est consommé comme tel, surtout par les enfants. La pulpe de ditax (verte et 
farineuse) est utilisée pour préparer des nectars, des marmelades et des sirops très appréciés par le consommateur sénégalais. La pulpe est également utilisée pour apaiser la toux au Sénégal et en Guinée [28]. Au Nigeria, les fruits sont consommés comme tonique et stimulant pour les voyages [37]. En Côte-d'Ivoire, ils sont utilisés en friction locale contre les maux de reins chroniques [38].

La graine présente également des caractéristiques très intéressantes de par sa composition. Elle est très riche en polyosides hydrosolubles non-amylacés $\left(59,8 \mathrm{~g} \cdot 100 \mathrm{~g}^{-1}\right)$, principalement des xyloglucanes [11, 3942]. Ainsi, les graines sont utilisées dans l'alimentation et en médecine traditionnelle. Au Nigeria, la farine de la graine est traditionnellement utilisée comme agent épaississant dans les préparations culinaires [11]. En Côte-d'Ivoire et au Burkina Faso, les graines sont utilisées dans la fermentation de la bière de millet [6]. Au Sénégal, en Guinée, au Liberia et au Nigeria, les téguments de la graine sont consommés en tant qu'aliment lors des famines [5, 11]. Dans le Nord du Nigeria, dans la médecine traditionnelle, les téguments de la graine sont utilisés pour traiter les cas d'empoisonnement aux flèches alors que, au Sénégal, les graines brûlées sont utilisées comme anti-moustique [6]. Les graines de Detarium senegalense peuvent également servir potentiellement de supplément diététique pour accroittre le contrôle de la glycémie [39-42]. C'est ainsi que, dans les zones urbaines du Nigeria, elle sont utilisées dans le traitement du diabète [11].

\section{Transformation du fruit}

\subsection{Aptitudes des fruits à la transformation}

La qualité de la pulpe de ditax obtenue dépend de l'état de maturité des fruits à la récolte, du mode de transformation primaire, mais aussi, en grande partie, de la provenance des fruits. Les fruits immatures ne sont pas transformables même avec des moyens mécaniques car la séparation de l'épicarpe du mésocarpe est impossible. Pour les fruits mûrs, l'épicarpe devient cas- sant quand on le presse avec les doigts. Les fruits bien mûrs sont faciles à triturer. Ils sont plus petits et très sucrés, surtout quand ils mûrissent sur l'arbre. Les fruits arrivent sur les marchés de Dakar encore immatures, mais au stade de récolte convenable, et subissent une maturation complémentaire. Après broyage et trituration des fruits, la couleur verte de la pulpe obtenue s'altère rapidement. Cette dégradation de la coloration verte est également observée dans les produits élaborés à partir de ditax surtout pour ceux ayant subi un traitement thermique. Dans les nectars, une séparation de phase survient en plus rapidement au cours du stockage, modifiant fortement l'aspect des produits [29].

\subsection{Transformation primaire du ditax}

La transformation primaire du ditax permet d'obtenir une purée raffinée qui après une transformation secondaire donne différents produits comme les nectars, les bases concentrées, les marmelades, les purées raffinées ou les pâtes séchées [29-31].

\subsubsection{Transformation artisanale}

Dans les concessions familiales et dans la plupart des unités de transformation, le décorticage et l'extraction de la pulpe se font manuellement à l'aide d'un pilon et d'un mortier. La plupart du temps, les fruits sont pilés avec l'épicarpe, mais ils peuvent être décortiqués manuellement pour une meilleure qualité de la purée. Cependant, le décorticage manuel est très fastidieux surtout lorsqu'il s'agit de traiter de grande quantité de fruits.

Après nettoyage, les fruits de maturité homogène sont pilés au mortier (figure O) donnant alors un mélange très pâteux et compact constitué de purée, de débris de coques et de noyaux. Du fait de la nature pulvérulente de la chair et du réseau fibreux insérant la pulpe, il est nécessaire d'ajouter de d'eau à la pulpe pâteuse obtenue afin de la délayer. La quantité d'eau à ajouter dépend du produit final à fabriquer. Par exemple, il faut ajouter (3 à 4) L d'eau par kg de mélange pour la préparation d'un nectar, alors qu'il faut $1 \mathrm{~L}$ d'eau pour $4 \mathrm{~kg}$ de mélange pour la fabrication des bases 
concentrées. Les noyaux sont ensuite malaxés pour enlever totalement la pulpe résiduelle encore entremêlée dans le réseau fibreux. La purée obtenue est alors tamisée grossièrement $(2 \mathrm{~mm})$ pour éliminer les noyaux, puis raffinée avec un tamis fin (1 mm) pour enlever les débris (coques, fibres fines, etc.).

\subsubsection{Décorticage et extraction mécaniques de la purée de Ditax}

Pour le décorticage et l'extraction mécaniques du ditax, les fruits, à raison de 9 à $10 \mathrm{~kg}$ par charge, sont triturés dans une parmentière munie d'un plateau et de parois abrasives (figure 7). Le plateau en tournant projette les fruits sur les parois rugueuses qui les décortiquent par abrasion. Une petite quantité d'eau est ajoutée pour permettre l'écoulement de la pulpe. La purée grossière composée de pulpe, de débris de coques cassées et de fibres est entraînée par l'eau introduite et recueillie dans une bassine au niveau de la partie inférieure de la parmentière tandis que les noyaux dépulpés sont retenus à l'intérieur. La pulpe est ensuite raffinée dans une passoire munie de plusieurs jeux de tamis. La purée raffinée collectée sert de base pour la fabrication des produits dérivés du ditax comme les nectars, les sirops, les marmelades ou les pâtes de fruits [29-31].

\subsubsection{Fabrication des produits à base de ditax}

A partir de la purée de ditax raffinée, divers produits peuvent être obtenus : du nectar, du sirop ou de la marmelade. La formulation du nectar consiste à ajouter $13 \%$ de sucre et $0,3 \%$ d'acide citrique à la purée raffinée diluée à $3{ }^{\circ} \mathrm{Brix}$, (figure 8). Pour la fabrication de la marmelade, il faut ajouter $45 \%$ de sucre et $0,3 \%$ d'acide citrique à la purée (55\%) diluée entre $10^{\circ} \mathrm{Brix}$ et $12^{\circ} \mathrm{Brix}$ [29-31].

\section{Aspects socio-économiques et statistiques de production du ditax au Sénégal}

Dans les zones de production du Sénégal, principalement dans la région de Fatick, le

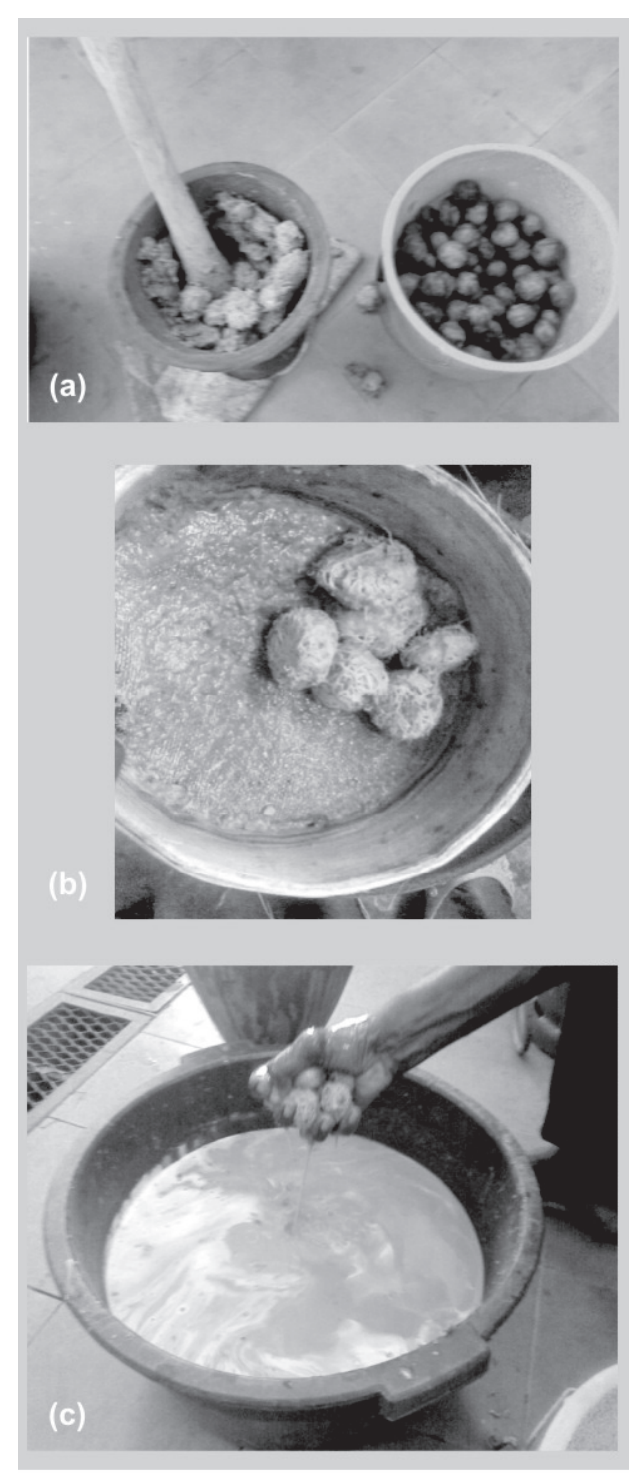

Figure 6.

Différentes étapes de la transformation artisanale du ditax (Detarium senegalense J. F. Gmel.) : décorticage et extraction de la pulpe au mortier (a), tamisage manuel (b) et purée diluée (c). ditax constitue un produit très important dans l'économie d'exploitation. Dans certaines zones, il constitue la principale source de revenus des populations. Dans les îles du Saloum par exemple, la totalité des frais de scolarisation des enfants est couverte par la vente directe des fruits [25]. De même, le ditax constitue la seconde source de recette des Eaux et Forêts après celle provenant de l'octroi des permis de chasse dans cette zone. La taxe est fixée par décret à $15 \mathrm{FCFA} \cdot \mathrm{kg}^{-1}$. Le poids homologué du panier est de $50 \mathrm{~kg}$ pour lequel, à Dakar, le prix de vente est compris entre (8 000 et 20 000) FCFA, soit 
Figure 7.

Extracteur mécanique de la pulpe de ditax (Detarium senegalense J. F. Gmel.).

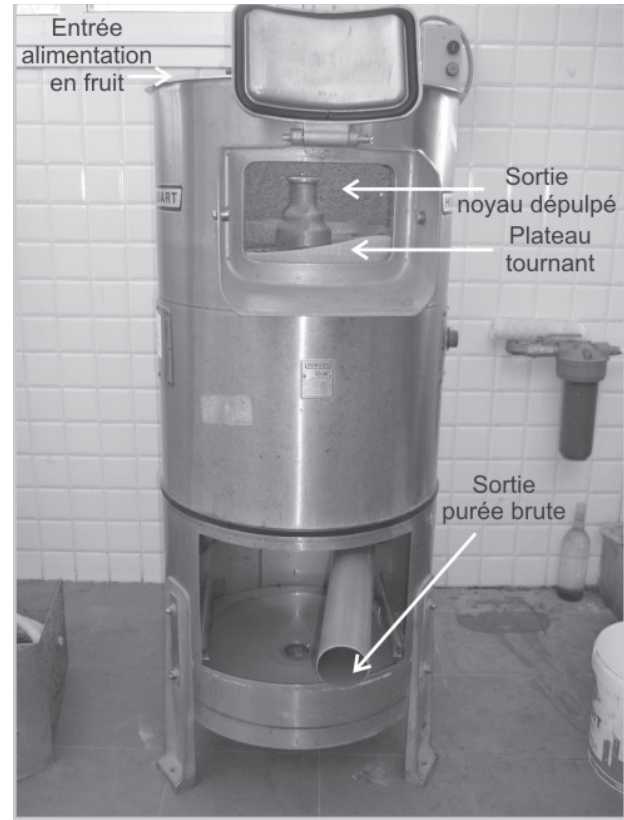

entre (160 et 400) FCFA $\cdot \mathrm{kg}^{-1}$. Les prix au détail varient entre (500 et 800) $\mathrm{FCFA} \cdot \mathrm{kg}^{-1}$ [7, 25, 43].

L'évolution de la production de ditax enregistrée au Sénégal a été globalement croissante de 1997 à 2007 (figure 9). Estimée à moins de 10 t en 1997, cette production est passée à près de 840 t en 2006 [43]. Cette augmentation serait liée au développement des infrastructures routières facilitant l'accès aux zones les plus reculées mais elle serait aussi due à l'arrêt des conflits en Casamance. Cependant, cette production officielle est largement inférieure à la production totale. En effet, ces données sont basées sur la production déclarée par les exploitants et enregistrée par les services d'inspections régionales des Eaux et Forêts. Elles ne considèrent pas les fruits autoconsommés et ne tiennent pas compte de la difficulté à distinguer les fruits provenant du Sénégal et ceux produits par les pays frontaliers voisins que sont la Gambie et les deux Guinées. Le niveau des pluies dans les différentes zones de production, l'accessibilité des zones et/ ou une variabilité entre les sujets selon les saisons expliqueraient également les fluctuations observées au niveau de cette production de ditax.

L'exploitation de Detarium senegalense comme ressource forestière limite le potentiel économique. Des essais de domestica- tion sont en cours de réalisation ce qui permettra à long terme d'une part d'augmenter la productivité, d'autre part d'envisager une valorisation industrielle [12]. La mise en place d'une filière de production devrait permettre de définir les modalités de commercialisation.

\section{Circuits de collectes et de distribution du ditax}

Lorsque les fruits de ditax arrivent à maturité, les commerçants ambulants appelés "bana-banas" convergent vers les zones de production pour acheter directement chez les populations rurales des bassines ou paniers de ditax. Dans la région de Fatick, la cueillette est réservée en priorité aux populations locales. Dans cette zone, des "Commissions Rurales Forêts" (commissions formées par les populations locales avec l'appui des services forestiers) sont chargées de la surveillance et de l'exploitation des produits de la forêt. Ce sont ces commissions qui fixent la date de démarrage de la cueillette des fruits. Elles sensibilisent les populations sur les modes d'exploitation du ditax en interdisant par exemple l'élagage comme mode de récolte des fruits car cette pratique affaiblit l'arbre [7]. Une fois la cueillette terminée, les populations déclarent les quantités de fruits auprès du service forestier. Les fruits sont ensuite acheminés vers Dakar, Pikine et vers d'autres centres urbains et zones touristiques. Le transport des fruits vers les zones de consommation est très difficile et très coûteux. En effet, à partir des îles du Sine-Saloum, le ditax est transporté par voie maritime puis par route ; à partir de la Casamance, le transport est assuré par de gros camions qui peuvent mettre une semaine avant de rejoindre, par la route, les grands marchés urbains entrâ̂nant ainsi d'énormes pertes post-récolte. Les "bana-banas " en provenance des zones de production revendent leurs produits aux grossistes installés aux marchés " syndicats " de Pikine, Tilène, Sandaga et au marché du port de Dakar. Les demi-grossistes et les petits commerçants viennent s'approvisionner chez les grossistes pour ensuite écouler les fruits sur les marchés de quartier. Les unités de transformation viennent également se ravitailler auprès des grossistes. 


\section{Conclusions}

Arbre caractéristique des îles du SineSaloum et de la forêt casamançaise, Detarium senegalense J.F. Gmel. (variété comestible) produit des fruits très appréciés par les populations locales sénégalaises. Le fruit, dont la pulpe est la principale partie valorisée, est caractérisé par une teneur en vitamine $\mathrm{C}$ très élevée supérieure à $1000 \mathrm{mg} \cdot 100 \mathrm{~g}^{-1}$ de pulpe fraîche. La pulpe verte et acidulée est généralement consommée à l'état frais ou sous forme de boissons, de marmelades, de sorbets et de sirops. Cependant, la boisson pose des problèmes de stabilité au cours des opérations de transformation et du stockage (dégradation de la couleur verte).

Le ditax reste malgré tout sous-exploité en dépit de ses caractéristiques nutritionnelles intéressantes. La caractérisation biochimique de la pulpe est très incomplète notamment en ce qui concerne sa composition en acides organiques, son profil glucidique, son pouvoir antioxydant et sa teneur en pigments.

La graine, de par sa composition et ses nombreuses utilisations aussi bien alimentaires que pharmaceutiques, mériterait une plus grande attention. La caractérisation physico-chimique et biochimique de ses différents constituants ainsi que l'étude de leurs propriétés sont des perspectives de recherche intéressantes à envisager.

Actuellement, le ditax est exploité à l'état spontané. Des recherches de domestication de l'arbre ont débuté pour raccourcir les délais de fructification et pour inciter son exploitation en verger. Cela devrait permettre d'augmenter considérablement la production en fruits et de régulariser l'approvisionnement en matière première des unités de transformations locales. Le ditax présente un potentiel commercial très significatif sur les marchés urbains locaux et régionaux ainsi que, surtout, à l'international. Grâce à son arôme, à sa couleur verte caractéristique et à sa teneur naturelle en vitamine $\mathrm{C}$, le nectar de ditax offre des perspectives de développement sur les marchés de niches à l'export.

Pour cela, la description des principaux éléments caractéristiques de la filière Deta-

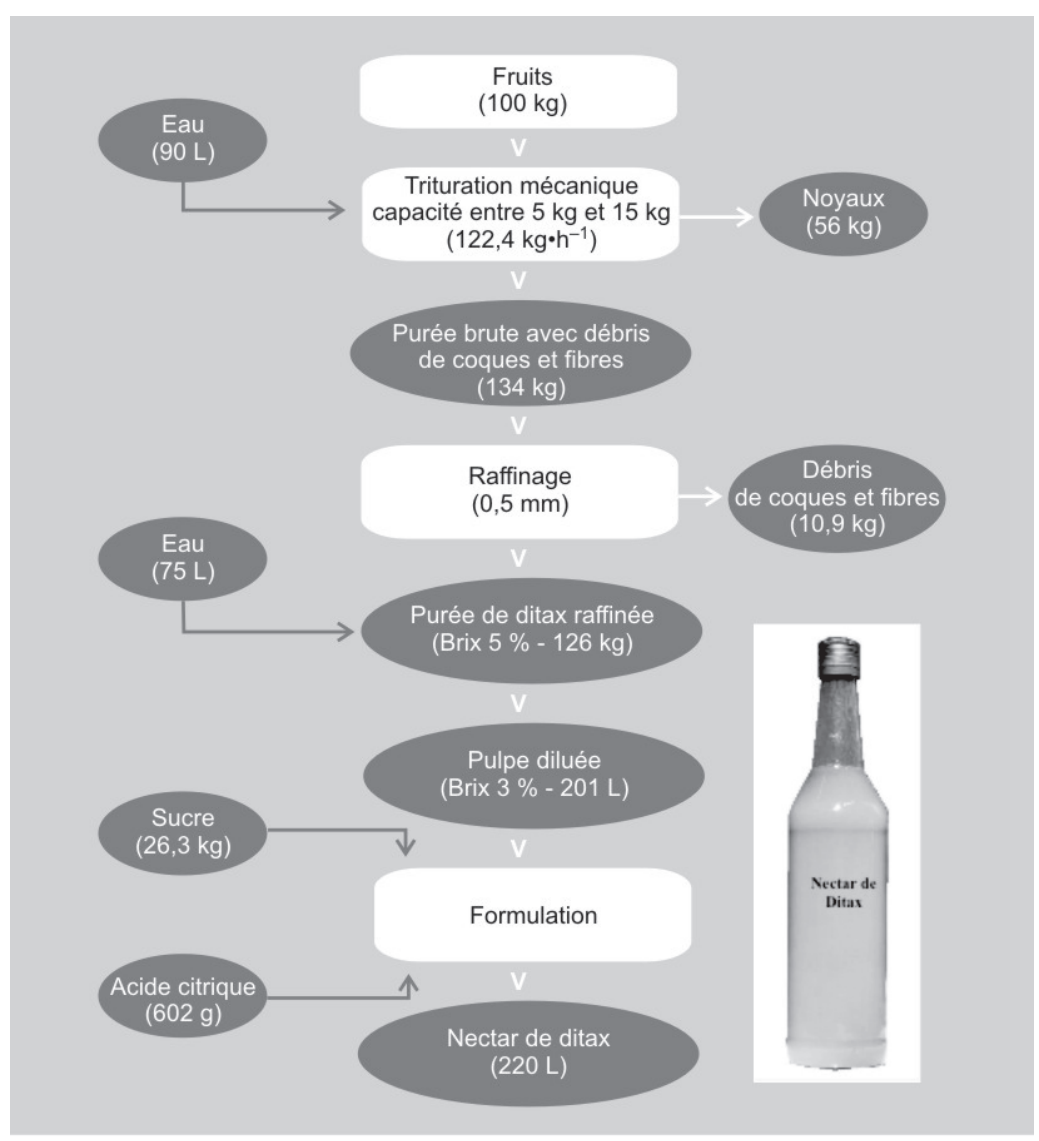

rium senegalense au Sénégal serait intéressante. Il s'agira de traiter tour à tour de la culture, des producteurs, des exploitants, des commerçants et des circuits de collecte, de distribution et de commercialisation, en passant par les opérations et les acteurs de la transformation.

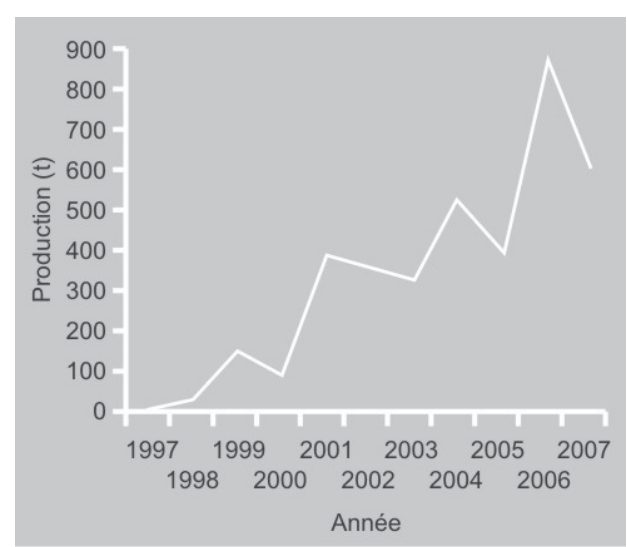

Figure 9.

Evolution de la production enregistrée de ditax de 1997 à 2007 au Sénégal [43]. nectar de ditax (Detarium senegalense J. F. Gmel.) standardisé par l'Institut de Technologie Alimentaire (ITA) de Dakar.
Diagramme de fabrication du 
Tableau III. Composition chimique (constituants pour $100 \mathrm{~g}$ de pulpe fraîche) de la pulpe de ditax (Detarium senegalense).

\begin{tabular}{|c|c|c|c|c|c|c|c|c|c|c|c|c|c|c|c|}
\hline Référence & $\begin{array}{c}\text { Fraction } \\
\text { comestible } \\
(\%)\end{array}$ & $\begin{array}{c}\text { Energie } \\
\text { (kJ) }\end{array}$ & $\begin{array}{c}\text { Eau } \\
\text { (g) }\end{array}$ & $\begin{array}{l}\text { Protéines } \\
\text { (g) }\end{array}$ & $\begin{array}{l}\text { Lipides } \\
\text { totaux } \\
\text { (g) }\end{array}$ & $\begin{array}{c}\text { Glucides } \\
\text { disponibles } \\
\text { (g) }\end{array}$ & $\begin{array}{c}\text { Fibres } \\
\text { brutes } \\
\text { (g) }\end{array}$ & $\begin{array}{l}\text { Calcium } \\
\text { (mg) }\end{array}$ & $\begin{array}{l}\text { Fer } \\
(\mathrm{mg})\end{array}$ & $\begin{array}{l}\text { Phosphore } \\
\text { (mg) }\end{array}$ & $\begin{array}{c}\text { Eq. } \beta \\
\text { carotene } \\
(\mathrm{mg})\end{array}$ & $\begin{array}{c}\text { Vitamine } \\
\text { C (mg) }\end{array}$ & $\begin{array}{l}\text { Thiamine } \\
\text { (mg) }\end{array}$ & $\begin{array}{l}\text { Riboflavine } \\
\text { (mg) }\end{array}$ & $\begin{array}{c}\text { Niacine } \\
\text { (mg) }\end{array}$ \\
\hline [30] & 47 & 484 & 66,9 & 1,9 & 0,4 & 27,3 & 2,3 & 27 & 2,8 & 48 & 165 & 1130 & 0,13 & 0,05 & 0,6 \\
\hline [10] & - & - & 73,8 & 2 & 0,4 & 29,7 & - & - & 3 & 49 & 132 & 1290 & 0,3 & - & - \\
\hline
\end{tabular}

Ainsi, la caractérisation complète du fruit comestible, l'évaluation du potentiel technologique et l'amélioration de la stabilité des produits dérivés sont des perspectives importantes pour mieux valoriser ce fruit et accroître les revenus des exploitants. La prise en compte de l'existence dans la nature d'une variété produisant des fruits toxiques implique également la recherche d'alcaloïdes et de dérivés glycosidiques cyanogènes dans ces fruits. Dans ce contexte, il serait très utile de mettre au point une méthode permettant de différencier facilement les fruits toxiques des fruits comestibles dans les zones de production de ditax concernées.

\section{Remerciements}

Les auteurs remercient le personnel de l'Institut de Technologie Alimentaire (ITA) de Dakar, particulièrement M. Babacar Dieng et Mme Mary Forster de l'Atelier Fruits et Légumes ; Mme Anta Faye Diallo et M. Pape Demba Camara du service de la documentation de l'ITA ; M. Ibrahima Ndiaye de la DEFCCS (Direction des Eaux, Forêts, Chasse et Conservation des Sols, Sénégal) et M. Amsatou Thiam de l'ISRA/CNRF (Centre National de Recherche Forestières de l'Institut Sénégalais de Recherches Agricoles) pour leur contribution à cette synthèse bibliographique.

\section{Références}

[1] De Jussieu A. L., Genera Plantarum - Secundum Ordines Naturales Disposita, Ed. Herissant \& Barrois, Paris, France, 1789, 365 p.

[2] Gmelin J.F., Systema Naturae per Regna Tria Naturae, secundum Classes, Ordines,
Genera, Species, cum Characteribus, Differentiis, Tomus II, $13 \mathrm{e}$ ed., Ed. Impensis Georg. Emanuel. Beer., 1791, 700 p.

[3] Lô M., Contribution à l'étude biosystématique de Detarium senegalense J.F. Gmel., Univ. Cheikh Anta Diop, Dép. Biol. Vég., Mém. DEA, Dakar, Sénégal, 1988, 91 p.

[4] Diatta W., Contribution à l'étude pharmacognosique des fruits de deux variétés de Detarium senegalense J.F. Gmel. récoltées en Basse Casamance, Univ. Cheikh Anta Diop, Fac. Méd. Pharm., Thèse, Dakar, Sénégal, 1995, $124 \mathrm{p}$.

[5] Arbonnier M., Arbres, arbustes et lianes de zones sèches d'Afrique de l'Ouest, 2e éd., CIRAD-MNHN, Paris, France, 2002, 541 p.

[6] Cavin A.L., Contribution à la connaissance taxonomique et chimique de fruits africains du genre Detarium (Fabaceae - Caesalpinioideae) : D. microcarpum Guill. et Perr. et des formes comestibles et toxiques de D. senegalense J.F. Gmel., Univ. Genève, Fac. Sci., Thèse, 2007, 231 p.

[7] Anon., Étude agroforestière de Detarium senegalense, ISRA/CNRF, Dakar, Sénégal, 2000, $18 \mathrm{p}$.

[8] Hutchinson J., Dalziel J.M. (revis. by Keay R.W.J.), Flora of West tropical Africa, vol I, part II, 2nd Ed., Whitefriars Press, London, U.K., 1958, 457 p.

[9] Berhaut J., Flore illustrée du Sénégal, Tome IV, Ed Clairafrique, Dakar, Sénégal, 1975, 625 p.

[10] Kerharo J., Adam J.G., La pharmacopée sénégalaise traditionnelle : plantes médicinales et toxiques, Ed. Vigot Frères, Paris, France, 1974, pp. 285-287.

[11] Haddad C., Fruitiers sauvages du Sénégal, Univ. Montpellier I, Thèse, Montpellier, France, 2000, 372 p.

[12] Anon., Domestication et valorisation de quelques fruitiers forestiers au Sénégal, 
Rapp. Final Proj. FNRAA, ISRA/CNRF, Dakar, Sénégal, 2003, $43 \mathrm{p}$.

[13] Guillemin J.A., Perrotet S., Richard A., Florae Senegambiae Tentamen, Tomus primus, Ed. APUD, Treuttel Würtz, Paris, France, 18301833, pp. 269-271.

[14] Imbert P., Teyssier J., Intoxication aiguë par ingestion de Ditakh. À propos de 8 observations, Med. Trop. 46 (1986) 79-83.

[15] Baillon H., Adansonia - Recueil périodique d'observations botaniques, Tome 6, Ed. E. Martinet, Paris, France, 1865-1866, pp. 200-202.

[16] Burgel P.R., Camara P., Collet Burgel C., Soko T.O., Hovette P., L'intoxication au Ditakh: une intoxication tropicale méconnue, 2 observations, Presse Med. 27 (1998) 1528.

[17] Berthelot P.G., N'Diaye M., Diatta B., Guerry B., Angel G., Saissy J.M., Acute intoxication after Ditakh fruit ingestion, Intensiv. Care Med. 26 (2000) 1587.

[18] Paris R., Moyse-Mignon H., A reputedly toxic Leguminosa of French West Africa "false detah" D. heudelotianum., Ann. Pharm. Fr. 5 (1947) 11-16.

[19] Sambuc M.C., Contribution à la flore et à la matière médicale de la Sénégambie, Ec. Sup. Pharm., Thèse, Montpellier, France, 1887, pp. 84-94.

[20] Heckel E., Schlagdenhauffen F., Sur les deux variétés de Detarium senegalense à fruits comestibles et à fruits amers au point de vue botanique et chimique, J. Pharm. Chim. 21 (1889) 401-414, 472-478.

[21] Adam F., Adam J.C., Hasselot N., Intoxication par ingestion de Ditakh (Sénégal), Med. Trop. 51 (1991) 455-458.

[22] Kerharo J., Adam J.G., Premier inventaire des plantes médicinales et toxiques de la Casamance, Ann. Pharm. Fr. 20 (1962) 726727.

[23] D'Almeida P.A., Trente-trois cas d'intoxication par les plantes observés au service de pédiatrie de l'hôpital principal de Dakar, Dép. Pharm., Hôpital Princ. Dakar, Thèse, Dakar, Sénégal, 1984, pp. 124-170.

[24] Aubreville A., Trochain J., Les espèces du genre Detarium (Légum. Césalpiniacées) en A.O.F., Bull. Soc. Bot. Fr. 84 (1937) 487-494.

[25] Diop N., Collecte de Ditax dans les zones inventoriées au niveau de la région de Fatick,
Amélioration de la compétitivité des boissons et nectars à base de fruits locaux au Sénégal, Proj. FNRAA-ITA, Rapp. Mission, Dakar, Sénégal, 2008, 5 p.

[26] Aubreville A., Flore forestière soudano-guinéenne: A.O.F Cameroun-A.E.F., Soc. Ed. Géogr. Marit. Colon., Paris, France, 1950, $523 \mathrm{p}$.

[27] Malgras D., Arbres et arbustes guérisseurs des savanes maliennes, Ed. Karthala / Agence de Coop. Cult. Techn., Paris, France, 1992, $479 \mathrm{p}$.

[28] Burkill H.M., The useful plants of West Tropical Africa, Vol. 3, Families J-L, 2nd Ed., Whitefriars Press, London, U.K., 1995, pp. 101-105.

[29] Anon., Valorisation d'un fruitier au Sénégal : le Ditax, Inst. Technol. Alim., Rapp. Act. Proj. SAFGRAD-ITA, ITA, Dakar, Sénegal, 1999, $22 \mathrm{p}$.

[30] Favier J.C., Ireland Ripert J., Laussucq C., Feinberg M., Table de composition des fruits exotiques, fruits de cueillette d'Afrique, (Répertoire général des aliments), ORSTOM, INRA, Tec \& Doc Lavoisier, Vol. 3, Paris, France, 1993, $243 \mathrm{p}$.

[31] Anon., Récolte et conservation du Ditax, in : Valorisation d'un fruitier au Sénégal: le Ditax, Atelier Prés. Résult. Rech., Inst. Technol. Alim., Proj. SAFGRAD-ITA, ITA, Dakar, Sénegal, 2001, $7 \mathrm{p}$.

[32] Auffret C., Présence de vitamine $C$ dans les fruits de Ditah, Maroc Méd. 207 (1948) 29.

[33] Toury J., Giorgi R., Favier J.C., Savina J.F., Aliments de l'Ouest Africain, Tables de composition, Ann. Nutr. Alim. 21 (2) (1967) 73127.

[34] Pousset J.L., Plantes médicinales africaines: possibilités de développement, Ellipses / Agence Coop. Cult. Techn., Tome I, Paris, France, 1989, 156 p.

[35] Diop P.A., Franck D., Grimm P., Hasselmann C., High-performance liquid chromatography determination of vitamin $C$ in fresh fruits from West Africa, J. Food Comp. Anal. 1 (1988) 265-269.

[36] Cisse M., Caractérisation de quelques fruits du Sénégal, stabilisation et concentration de jus de fruits tropicaux par des techniques membranaires, Univ. Cheikh Anta Diop, Thèse, Dakar, Sénégal, 2007, 210 p.

[37] Irvine F.R., Woody plants of Ghana, with special reference to their uses, Oxf. Univ. Press., London, U.K., 1961, XCV + 868 p. 
[38] Kheraro J., Bouquet A., Plantes médicinales et toxiques de la Côte d'Ivoire - Haute Volta, Ed. Vigot Frères, Paris, France, 1950, 296 p.

[39] Ellis P.R., Rayment P., Wang Q., A physicochemical perspective of plant polysaccharides in relation to glucose absorption, insulin secretion and the entero-insular axis, Proc. Nutr. Soc. 55 (1996) 881-898.

[40] Wang Q., Ellis P.R., Ross-Murphy S.B., Burchard W., Solution characteristics of the xyloglucan extracted from Detarium senegalense Gmelin., Carbohydr. Polym. 33 (1997) 115-124.
[41] Wang Q., Ellis P.R., Ross-Murphy S.B., Grant Reid J.S., A new polysaccharide from a traditional Nigerian plant food: Detarium senegalense Gmelin., Carbohydr. Res. 284 (2) (1997) 229-239.

[42] Onyechi U.A., Judd P.A., Ellis P.R., African plant foods rich in non-starch polysaccharides reduce postprandial blood glucose and insulin concentrations in healthy human subjects, Br. J. Nutr. 80 (1998) 419-428.

[43] Anon., Statistiques officielles de production du Ditax, DEFCCS, Div. Aménag. Prod. For., Dakar, Sénégal, 2008.

\section{El ditax (Detarium senegalense J.F. Gmel.) : principales características y usos en Senegal.}

Resumen - Botánica. Natural de África tropical, Detarium senegalense J.F. Gmel. pertenece a la familia de los Caesalpiniaceae y al género Detarium Juss. Es un gran árbol multicaule de (15 en 40) $\mathrm{m}$ de alto. Sus hojas son compuestas paripinnadas con cinco a seis pares de foliolos opuestos dispuestos de manera alterna. Distribución. D. senegalense se encuentra alrededor de los lindes del bosque denso húmedo, en las regiones costeras, septentrionales y en la zona sudano-guineana. Designado por el término "ditax" en Wolof, D. senegalense forma parte de las especies frutales forestales más importantes en la economía de explotación en Senegal. Crece de manera salvaje en las islas del Sine-Salum y en Casamance. Composición y transformación. La fruta es caracterizada por su pulpa verde muy rica en vitamina C (más de $1 \mathrm{~g} \cdot 100 \mathrm{~g}^{-1}$ ). Es una fruta muy popular y muy consumida en Senegal principalmente en forma de bebida, mermelada, sorbete o en el estado fresco. La fruta posee también virtudes terapéuticas como contra la tos, los males de riñones y contra la lepra. Algunas variedades de D. senegalense producen frutas tóxicas pero no se identificaron aún los compuestos responsables de la toxicidad. Actualmente no hay ningún método rigoroso que permite diferenciar las frutas comestibles de las frutas tóxicas. Conclusión. A pesar de sus potencialidades nutricionales y organolépticas, el ditax no se explota lo suficiente. Por lo tanto, la caracterización bioquímica completa de las frutas, la evaluación del potencial tecnológico, el desarrollo de métodos de detección rápida de las frutas tóxicas y la mejora de la estabilidad de los productos transformados a base de Ditax son objetivos de investigación muy interesantes para valorizar mejor esta fruta rica en vitamina $\mathrm{C}$. El cultivo controlado de la planta merecería también es un factor a tener en cuenta para el desarrollo de la producción.

Senegal / Detarium senegalense / frutas / composición aproximada / usos tradicionales / toxicidad por ingestión / ácido ascórbico 